\title{
A quest towards personalised medicine for grade II meningiomas-will need to zoom in
}

\author{
Thomas Santarius $^{1} \cdot$ Michael D. Jenkinson ${ }^{2} \cdot$ Ramez W. Kirollos ${ }^{1,3}$
}

Received: 3 March 2016 / Accepted: 9 March 2016/Published online: 28 March 2016

(C) Springer-Verlag Wien 2016

Champeaux and Dunn [2] have analysed the outcomes of grade II meningiomas (atypical, clear cell and choroid) resected in the Southern General Hospital in Glasgow between January 2000 and August 2015. The authors first identified cases that were classified as grade 2 according to the classification used at the time of surgery. The histological reports (not the histological slides themselves) were carefully reviewed and cases from before 2007 were reclassified according to the WHO 2007 classification of the tumours of the central nervous system [10] (further referred to as WHO 2007 classification). In the end, 206 cases were included in the study and only two patients were lost to follow-up. The median follow-up was 4.1 years, with the interquartile range of 1.6-7.3 years.

Overall survival probabilities at 1,5 and 10 years were respectively: 95,84 and $73 \%$ - easy numbers to remember. Even based on the relatively low numbers, the $95 \%$-confidence intervals are fairly tight: 93-98, 78-90, and 65-82\%, respectively. When the 5 -year overall survival of the Glasgow cohort is viewed in the context of other series (Table 3) [2], it seems that 5-year survival of surgically treated atypical meningiomas ranges from approximately $75-90 \%$ (an apparent outlier series put aside).

Thomas Santarius

thomas.santarius@cantab.net

1 Department of Neurosurgery, Addenbrooke's Hospital, University of Cambridge, Hills Road, Box 166, Cambridge, UK

2 Department of Neurosurgery, Walton Centre for Neurology and Neurosurgery, University of Liverpool, Liverpool, UK

3 Khu Teck Puat Hospital, National University of Singapore, Singapore, Singapore
Univariate analysis of the cohort revealed age, Simpson grade, presence of brain invasion, venous sinus invasion, tumour volume and reoperation to be associated with survival. These variables were then entered into the Cox multivariate regression model, which identified age (62 years and lower), Simpson resection grade (grades 1-3 versus 4-5) and absence of brain invasion as predictors of longer overall survival.

Although questioned by some [14], Simpson grade remains a powerful determinant of prognosis for meningiomas, including atypical meningiomas. In fact, along with our anecdotal observation, Gousias et al. [5] found in their series of 901 consecutive meningioma resections that the influence of Simpson grade on progression-free survival (PFS) was even greater in grade II and III than grade I meningiomas.

Radiotherapy was not a factor predictive of survival, even in the univariate model. The specific role of adjuvant radiotherapy for individual tumours remains unclear and has not been rigorously assessed in the existing literature [11, 12]. For grade II meningioma, two phase-2 dose-escalation studies by the EORTC and RTOG are due to report soon and the upcoming ROAM/EORTC 1308 trial will address the role of early radiotherapy following gross total resection [6]. Not surprisingly, at least for now, meningiomas remain a "surgical" disease with regards to their treatment.

Brain invasion was the basis of the last modification of the meningioma grading published in the in the 2007 edition of the WHO classification of the tumours of the central nervous system [10]. In the 1993 classification, when atypical meningioma was first introduced, brain invasion was a feature that automatically upgraded the tumour to an anaplastic category [8]. The next edition, the $2000 \mathrm{WHO}$ classification [7], saw major changes introduced to the grading of meningiomas. The previously vague histological descriptions were tightened up with explicitly defined mitotic rates for each grade. Brain invasion in the context of otherwise benign histological 
appearance itself defines the tumour as grade II, thus broadening the inclusion criteria for grade II. Indeed, this has been reflected in the increase in the incidence of grade II and decrease of grade III meningiomas [9]. Backer-Grøndahl et al. [1] state in their paper on 196 consecutive meningiomas that if they were to use the $2000 \mathrm{WHO}$ classification, the proportion of their grade II tumours would have been $26 \%$ instead of $30 \%$ as per the 2007 WHO classification.

Despite progressive improvements in the classification of meningiomas, we know from our clinical observations that atypical meningiomas are far from being homogeneous in their natural history and response to treatment. The study by Champeaux and Dunn has played its part in contributing to better understanding of grade II meningiomas and has identified a number of factors affecting outcome [2]. However, there is still a need for carefully designed and meticulously executed studies, ideally where genotype and high-quality phenotype data are captured simultaneously. Because cancer is a genetic disease [13], and meningioma is a type of cancer, understanding of its genetic landscape will form the bases of future sub-classifications, similarly to what we have been witnessing in haematological conditions, breast cancer, paediatric brain tumours, etc. [3, 4]. It is now fashionable to talk about molecular markers, but undoubtedly phenotypical characteristics, both histological and clinical, will continue to play a vital role. Similarly to histological sampling, molecular sampling is susceptible to a potential sampling error. This probably plays a lesser role than in gliomas, for example, given the lower incidence of malignant progression and assuming less intra-tumoural heterogeneity of meningiomas, but needs to be understood and taken into account.

Detailed and accurate molecular and clinical characterisation of the tumour forms the basis of so-called personalised medicine, another fashionable term and a goal being currently pursued in every branch of medicine. However, in the quest of pursuing this goal we must not forget that our role is to treat a patient with a disease rather than just the disease, as finely and precisely as it may be characterised. Otherwise we may, paradoxically, be in danger of moving away from truly personalised (tailored) medicine back to the 'small/medium/ large' era.

\section{References}

1. Backer-Grøndahl T, Moen BH, Torp SH (2012) The histopathological spectrum of human meningiomas. Int J Clin Exp Pathol 5(3): 231-242

2. Champeaux C, Dunn L (2016) World Health Organization grade II meningiomas. A 10-year retrospective study on overall survival and associated prognostic factors. Acta Neurochir (Wein). doi:10.1007/ s00701-016-2771-y

3. Curtis C, Shah SP, Chin S-F et al (2012) The genomic and transcriptomic architecture of 2,000 breast tumours reveals novel subgroups. Nature 486(7403):346-352

4. Gajjar A, Pfister SM, Taylor MD, Gilbertson RJ (2014) Molecular insights into pediatric brain tumors have the potential to transform therapy. Clin Cancer Res 20(22):5630-5640

5. Gousias K, Schramm J, Simon M (2016) The Simpson grading revisited: aggressive surgery and its place in modern meningioma management. J Neurosurg 1-10

6. Jenkinson MD, Javadpour M, Haylock BJ et al (2015) The ROAM/ EORTC-1308 trial: radiation versus observation following surgical resection of atypical meningioma: study protocol for a randomised controlled trial. Trials 16:519

7. Kleihues P, Cavenee WK (2000) World Health Organization classification of tumours - pathology and genetics. Tumours of the nervous system. IARC Press, Lyon

8. Kleihues P, Burger PC, Scheithauer BW (1993) Histological typing of tumours of the central nevous system. Springer, Berlin

9. Kshettry VR, Ostrom QT, Kruchko C, Al-Mefty O, Barnett GH, Barnholtz-Sloan JS (2015) Descriptive epidemiology of World Health Organization grades II and III intracranial meningiomas in the United States. Neuro-Oncology 17(8):1166-1173

10. Louis DN, Ohgaki H, Wiestler OD, Cavenee WK (eds) (2007) WHO classification of tumours of the central nervous system. IARC, Lyon

11. Marcus HJ, Price SJ, Wilby M, Santarius T, Kirollos RW (2008) Radiotherapy as an adjuvant in the management of intracranial meningiomas: are we practising evidence-based medicine? $\mathrm{Br} \mathrm{J}$ Neurosurg 22(4):520-528

12. Rogers L, Barani I, Chamberlain M, Kaley TJ, McDermott M, Raizer J, Schiff D, Weber DC, Wen PY, Vogelbaum MA (2014) Meningiomas: knowledge base, treatment outcomes, and uncertainties. A RANO review. J Neurosurg 1-20

13. Stratton MR, Campbell PJ, Futreal PA (2009) The cancer genome. Nature 458(7239):719-724

14. Sughrue ME, Kane AJ, Shangari G, Rutkowski MJ, McDermott MW, Berger MS, Parsa AT (2010) The relevance of Simpson Grade I and II resection in modern neurosurgical treatment of World Health Organization Grade I meningiomas. J Neurosurg 113(5):1029-1035 